\title{
The curvature of the endotracheal tube exacerbated postoperative sore throat and hoarseness.
}

\author{
Musashi Yahagi, MD, Tsuyoshi Satsumae, MD, Makoto Tanaka, MD \\ Department of Anesthesiology, University of Tsukuba
}

\begin{abstract}
Background: We examined whether the two supporting methods of endotracheal tube (ETT) affected the frequency and severity of postoperative sore throat and hoarseness (POSTH). We hypothesized that supporting ETT with unsuitable curvature would increase the frequency of POSTH compared to with natural curvature.

Methods: Eighty-nine patients scheduled to undergo general anesthesia were studied. In the Natural group ( $n=45)$, ETT was connected to the breathing circuit with keeping natural curvature. In the Curved group $(n=44)$, ETT was bended 90 degrees to the right and connected to the breathing circuit. To evaluate the severity of POSTH, Stout's 4-point severity score was used. A blinded anesthesiologist recorded the score before leaving the operating room, on the day of surgery ( 2 to 8 hours after surgery) and the next day of surgery (24 to 36 hours after surgery).
\end{abstract}

Results: The overall incidence of postoperative sore throat (POST) was higher in the Curved group than the Natural group (82\% vs $40 \%$; relative risk $=2.16,95 \%$ confidence interval: $1.48-3.15 ; p<0.001)$. The overall incidence of postoperative hoarseness was higher in the Curved group than the Natural group $(61 \%$ vs $31 \%$; relative risk $=1.97$, $95 \%$ confidence interval: $1.20-3.23 ; p=0.004)$. The POST severity scores were higher in the Curved group on the day of surgery ( $<<0.001)$ and the next day of surgery $(p<0.001)$.

Conclusion: POSTH aggravates when the tracheal tube is fixed with being unnaturally bent. To reduce the incidence and severity of POSTH, ETT should be appropriately supported without adding curvature.

【Introduction】

Postoperative sore throat and hoarseness (POSTH) is one of the complications of tracheal intubation during general anesthesia, which reduce patient satisfaction. El-Boghdadly et al. clarified the postoperative risk factors and the preventive method of POST in adults and children in a systematic review. Ref) This study was conducted in accordance with a protocol that aggressively excludes factors responsible for POSTH. Generally, the endotracheal tube (ETT) is connected to the respiratory circuit with keeping natural curvature during general anesthesia. However, when the position of ETT is restricted in order to secure a clean field, we have to curve ETT artificially. In this study, we examined whether the two supporting methods of ETT affected the frequency and severity of postoperative sore throat (POST) and hoarseness (POH).

【Methods】

Institutional review board approval and written informed consent were obtained. One hundred adult patients, ASA physical status I or II, were divided into two groups. In the Natural group, ETT was connected to the breathing circuit (Figure). After anesthetic induction, trachea was intubated using $\mathrm{McGrath}^{\mathrm{TM}}$. A Parker Flex-Tip ${ }^{\mathrm{TM}}$ endotracheal tube (internal diameter: $7.0 \mathrm{~mm}$ ) was used. Seal pressure of a cuff was adjusted to $20 \mathrm{~cm} \mathrm{H}_{2} \mathrm{O}$. After the operation finished, patient's trachea was extubated. To evaluate the severity of POST, the sore throat score $(0=$ none, $1=$ less severe than with a cold, $2=$ similar to that not with a cold, $3=$ more severe than with cold) was used. To evaluate the severity of $\mathrm{POH}$, the hoarseness score $(0=$ none, $1=$ noted by patient, 2 $=$ obvious to observer, $3=$ aphonia) was used. A blinded anesthesiologist recorded these scores before leaving the operating room, on the day of surgery ( 2 to 8 hours after surgery) and the next day of surgery ( 24 to 36 hours after surgery).

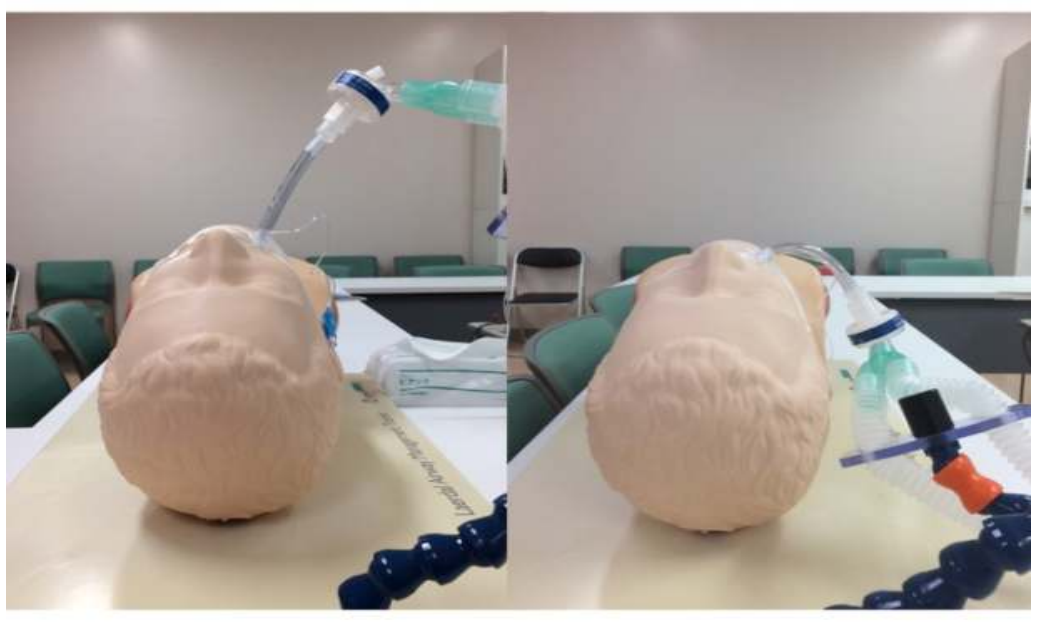

【Figure】

The model on the left is the Natural group, right is the Curved group.

Reference

El-Boghdadly K, Bailey CR, Wiles MD. Postoperative sore throat: a systematic review. Anaesthesia 2016; 71: 706-17
【Results】

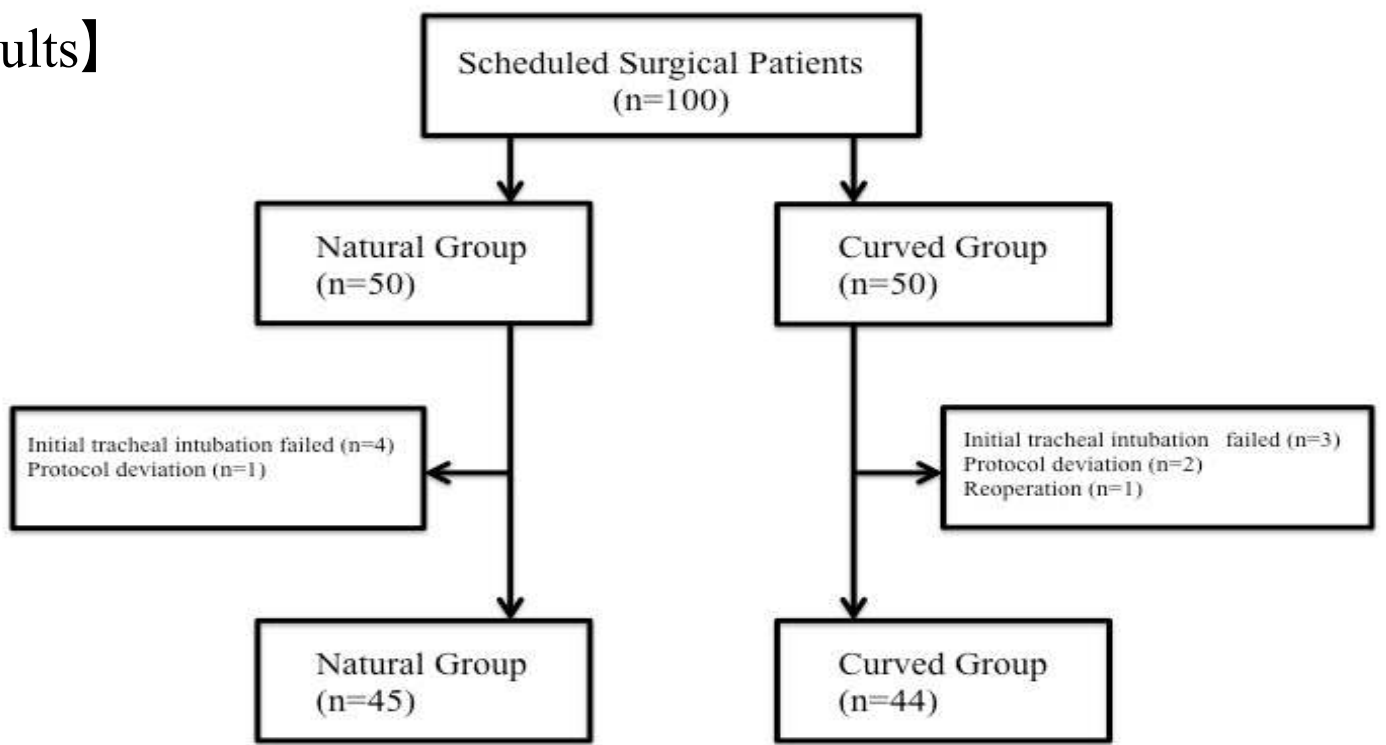

【Figure 2】 Flow diagram for patient inclusion and exclusion

\begin{tabular}{|l|r|r|r|r|r|}
\hline \multicolumn{1}{|c|}{ POST } & Natural & Curved & Relative risk & $95 \%$ confidence interval & p value \\
\hline Overall & $18(40.0)$ & $36(82.0)$ & 2.16 & $1.48-3.15$ & $<\mathbf{0 . 0 0 1 ^ { \mathrm { F } }}$ \\
\hline Before leaving the operating room & $12(26.7)$ & $14(31.8)$ & 1.19 & $0.60-2.18$ & $0.65^{\mathrm{F}}$ \\
\hline The day of surgery & $13(28.9)$ & $17(38.6)$ & 1.34 & $0.74-2.41$ & $0.38^{\mathrm{F}}$ \\
\hline The next day of surgery & $8(17.8)$ & $24(54.5)$ & 3.07 & $1.55-6.08$ & $<\mathbf{0 . 0 0 1}$ \\
\hline \multicolumn{7}{|c|}{ POH } & \multicolumn{5}{|c|}{} \\
\hline Overall & $14(31.1)$ & $27(61.4)$ & 1.97 & \multicolumn{1}{|c|}{$1.20-3.23$} & $\mathbf{0 . 0 0 4}$ \\
\hline Before leaving the operating room & $15(33.3)$ & $23(52.3)$ & 1.57 & $0.95-2.58$ & $0.05^{\mathrm{F}}$ \\
\hline The day of surgery & $6(13.3)$ & $24(54.5)$ & 4.09 & $1.85-9.93$ & $0.06^{\mathrm{F}}$ \\
\hline The next day of surgery & $18(40.0)$ & $13(29.5)$ & 0.74 & $0.41-1.32$ & $0.07^{\mathrm{F}}$ \\
\hline
\end{tabular}

【Table 1】 Frequency of POSTH F: Fisher's exact test

\begin{tabular}{|c|c|c|c|c|c|c|}
\hline & \multicolumn{5}{|c|}{ Severity Score } & \multirow[t]{2}{*}{$\mathrm{p}$ value } \\
\hline & Score 0 & Score 1 & Score 2 & Score 3 & Median [IQR] & \\
\hline POST & \multicolumn{6}{|c|}{ Natural / Curved } \\
\hline Before leaving the operating room & $33 / 30$ & $10 / 10$ & $2 / 2$ & $0 / 2$ & $0[0-2] / 0[0-3]$ & $0.52^{\wedge}$ \\
\hline The day of surgery & $32 / 27$ & $9 / 12$ & $4 / 5$ & $0 / 0$ & $0[0-2] / 1[0-2]$ & $<0.001^{\vee}$ \\
\hline The next day of surgery & $37 / 20$ & $7 / 22$ & $1 / 2$ & $0 / 0$ & $0[0-2] / 0[0-2]$ & $<0.001^{\vee}$ \\
\hline POH & \multicolumn{6}{|c|}{ Natural / Curved } \\
\hline Before leaving the operating room & $31 / 21$ & $7 / 9$ & $7 / 13$ & $0 / 1$ & $0[0-2] / 1[0-3]$ & $0.03^{3}$ \\
\hline The day of surgery & $30 / 30$ & $12 / 17$ & $3 / 7$ & $0 / 0$ & $0[0-2] / 1[0-2]$ & $0.04^{\mathrm{N}}$ \\
\hline The next day of surgery & $39 / 31$ & $4 / 10$ & $2 / 3$ & $0 / 0$ & $0[0-2] / 0[0-2]$ & 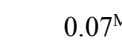 \\
\hline
\end{tabular}

【Table 2】 Stout's severity score of POSTH M. Mann-Whitney U test

【Conclusion】

POSTH aggravates when the tracheal tube is fixed with being unnaturally bent. If ETT is bent during general anesthesia, the risk of POSTH increases. To reduce the incidence and severity of POSTH, ETT should be appropriately supported without adding curvature. 